\title{
uvby photometry of 33 Tauri, HD 50169, and HR 7786 and an assessment of FCAPT comparison stars $^{\star}$
}

\author{
S.J. Adelman, C.-L.M. Pi, and K.E. Rayle \\ Department of Physics, The Citadel, 171 Moultrie Street, Charleston, SC 29409, U.S.A. \\ e-mail: adelmans@citadel.edu
}

Received April 15; accepted May 25, 1998

\begin{abstract}
Differential Strömgren uvby photometric observations from the Four College Automated Photoelectric Telescope are presented for the ellipsoidal variable 33 Tauri, and the magnetic Chemically Peculiar stars HD 50169 and HR 7786. We confirm that Hube's period of 2.975272 days for 33 Tau is correct. HD 50169 is a constant MCP star. HR 7786 is a large amplitude class member confirming Winzer's discovery, but its period is 8.5297 days. Its comparison star, HR 7721, a spectroscopic binary B7 $V$ star, is variable with an amplitude of order $0.1 \mathrm{mag}$, but of indeterminant period. In addition Hipparcos photometry of the comparison and check stars for early type stars whose photometry is being obtained with the FCAPT is reviewed to ascertain the stability of these stars.
\end{abstract}

Key words: stars: individual: 33 Tau; HR 7786; HD 50169 - stars: chemically peculiar

\section{Introduction}

Differential Strömgren photometry of three early type stars from the six years (September 1990 - July 1996) of the $0.75-\mathrm{m}$ Four College Automated Photoelectric Telescope (FCAPT) on Mt. Hopkins, AZ and from the first year after the telescope was moved to nearby Washington Camp, AZ (October 1966 - July 1997) are examined. After the dark count, the telescope measured

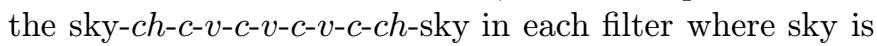
a reading of the sky, ch that of the check star, $c$ that of the comparison star, and $v$ that of the variable star. Table 1 contains group (a variable along with two supposedly non-variable stars, the comparison and check, against

Send offprint requests to: S.J. Adelman

* Tables 2-5 are only available in electronic form at the CDS via anonymous ftp to cdsarc.u-strasbg.fr (130.79.128.5) or via http://cdsweb.u-strasbg.fr/Abstract.html
Table 1. Photometric groups

\begin{tabular}{cllll}
\hline HD Number & Star Name & Type & $V$ & Spectral Type \\
& & & & \\
24769 & 33 Tau & $v$ & 6.06 & B9.5 IV \\
23753 & HR 1172 & $c$ & 5.45 & B8 V \\
24740 & 32 Tau & $c h$ & 5.63 & F2 IV \\
& & & & \\
50169 & BD $-01^{\circ} 1414$ & $v$ & 9.06 & A4p \\
50040 & BD $-01^{\circ}$ & $c$ & 8.36 & A0 \\
50405 & BD $-01^{\circ} 1428$ & $c h$ & 9.13 & A0 \\
& & & & \\
193722 & HR 7786 & $v$ & 6.50 & B9pSi \\
192514 & 30 Cyg & $c$ & 4.83 & A5 IIIn \\
192776 & HR 7721 & $c h$ & 6.92 & B7 V \\
\hline
\end{tabular}

which the brightness of the variable is compared) information (Hoffleit 1982). Tables 2-4 present the data and their yearly means and standard deviations. Corrections were not made for neutral density filter differences among the stars of each group. The comparison and check stars were chosen from supposedly non-variable stars in the vicinity of the variable on the sky that had similar $V$ magnitudes and $B-V$ colors. The standard deviations in Tables $2-4$ of the check-comparison star differences indicate that these stars are constant at least for the period when they were observed except for HR 7721.

For each group we plotted each variable's data using the best published period to see if the data approximately confirmed this period. Then we used the Scargle periodogram (Scargle 1982; Horne \& Baliunas 1986) and considered our data and those of other observers separately in calculating periodograms. If the periodograms confirmed the published period, then we adjusted the period to make all data coincide as well as possible in phase. 
33 Tauri
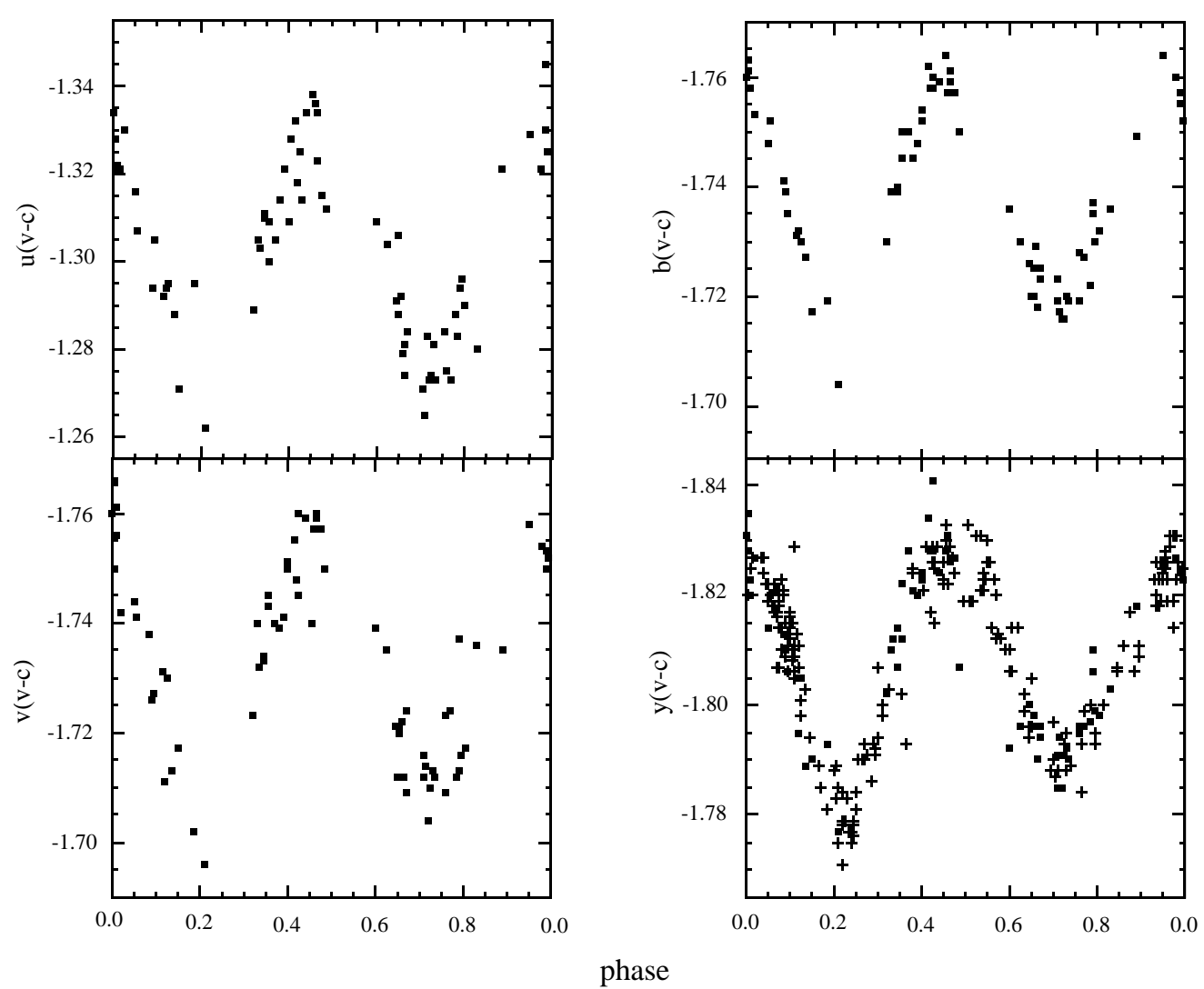

Fig. 1. Photometry of 33 Tau plotted according to the phase of the radial velocity maximum from Hube's ephemeris: HJD $2430000.626+(2.975272 \pm 0.000004) E$. The closed squares are FCAPT values from this paper and the crosses in the $y$ panel are the $V$ data of Gulliver et al. transformed to the FCAPT system

\section{33 Tau}

Winzer (1974) mistakenly thought that 33 Tau $(=\mathrm{HD} 24769=\mathrm{HR} 1221)$ was a magnetic $\mathrm{CP}$ star and found a period of 1.48 days. Instead (Morris 1985) it is a close binary star, an ellipsoidal variable. The photometric variations show two maxima and minima each orbital revolution with the two minima coinciding with the time of conjunction. Hube (1981) derived the orbital elements from radial velocity measurements and gave as the ephemerides for the phase of the velocity maximum $=$ HJD $2430000.626+(2.975272 \pm 0.000004)$ $E$. Gulliver et al. (1985) obtained $U B V$ photometry and modeled the system.

Forty-three and 38 sets of Strömgren observations were obtained during the 1995-96 and 1996-97 observing seasons, respectively. An examination of the $y$ photometry using the Scargle periodogram suggests a frequency of 0.3305 per day (a period of 3.0266 days) or its alias of 0.6695 per day. A periodogram analysis of the $V$ data of Gulliver et al. (1985) to our surprise also gave the same results. But that of the radial velocities confirms Hube's period. The 3.0266 day period results in a light curve with a single maximum and a single minimum and a radial velocity curve which is not easily interpreted.

Figure 1 shows our FCAPT data plotted with Hube's ephemeris. Our light curves look like those of Gulliver et al. We include their $V$ values in our $y$ plot after we renormalized them to our comparison star. We did not adjust the initial epoch as for three of the four light curves it is satisfactory. The light curves show differences between the first and second halves of the period. The amplitudes are about $0.07 \mathrm{mag}$ in $u, 0.06 \mathrm{mag}$ in $v, 0.05 \mathrm{mag}$ in $b$, and $0.06 \mathrm{mag}$ in $y$ which compare with Gulliver et al.'s $0.07 \mathrm{mag}$ in $U, 0.055 \mathrm{mag}$ in $B$, and $0.05 \mathrm{mag}$ in $V$. Additional uvby values near the extrema are needed to refine these values. That our $y$ values fall on top on their adjusted $V$ values using Hube's period indicates that his period error estimate should be reduced by a factor of 4 .

\section{HD 50169}

Mathys \& Hubrig (1997) note that Mathys et al. (1997) indicated that HD $50169\left(=\mathrm{BD}-1^{\circ} 1414=\right.$ Brewer's star) (Brewer 1953) had a period of rotation longer than 4 
HR 7786
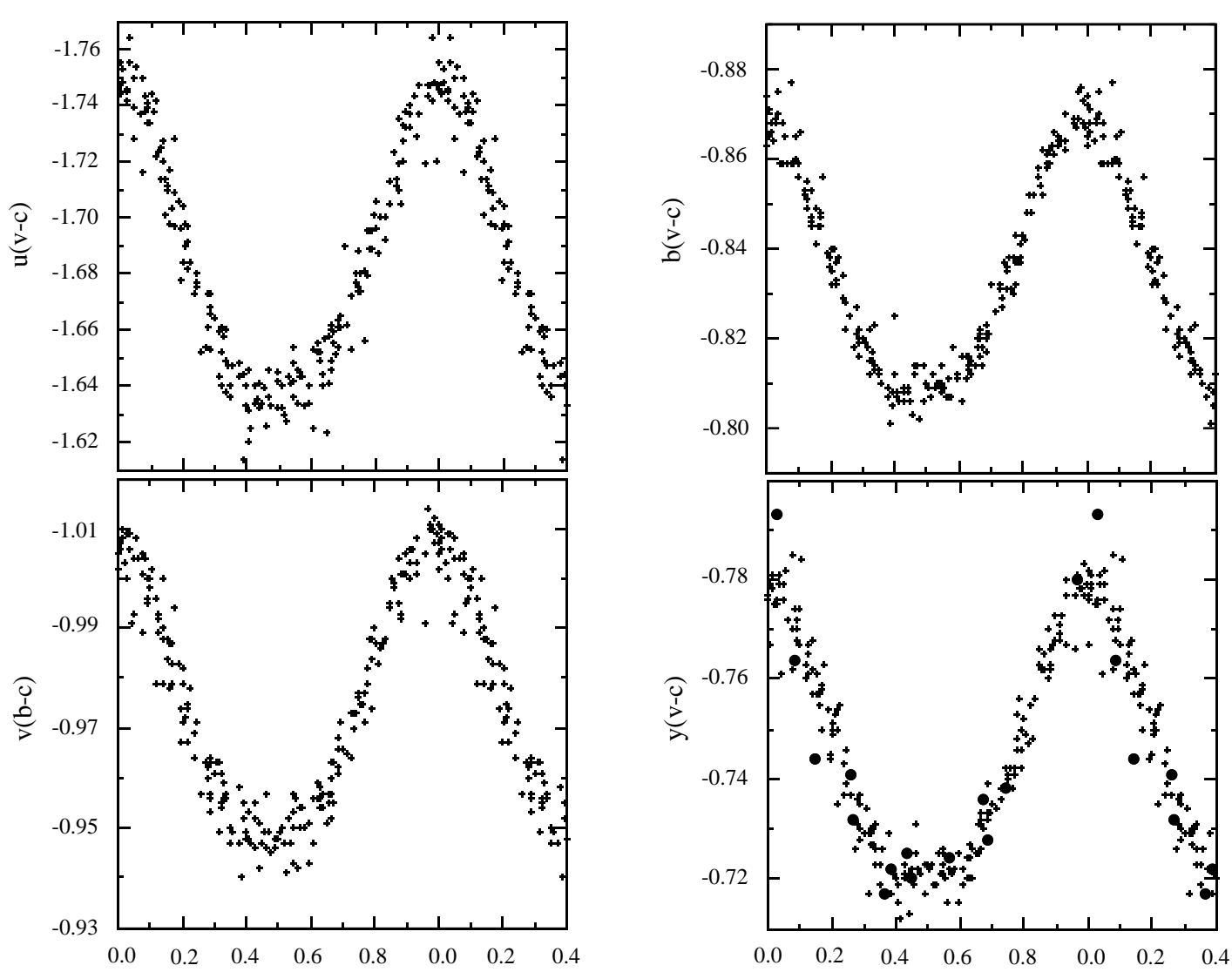

phase

Fig. 2. Photometry of HR 7786 plotted according to this paper's ephemeris. The FCAPT observations are indicated as plus signs and Winzer's $V$ magnitudes transformed to $y$ as closed circles

years from measurements of the mean magnetic field modulus. This is consistent with Babcock (1958)'s observation of a slow increase in the mean magnetic modulus over a period of three years. Photometric studies by Hensberge et al. (1981) and Heck et al. (1987) concluded this star was probably constant, but Heck et al. (1977) noted some short term variations. We obtained 68 sets of Strömgen observations in 1996-97 and 32 sets in 1997-98. The standard deviations of the means show that HD 51059 is constant as well as it comparison and check stars. Hipparcos photometry (see Sect. 5) confirms that this star is apparently non-variable.

\section{HR 7786 and HR 7721}

Winzer (1974) discovered HR 7786 (= HD $193722=$ V1584 Cyg) to be a large amplitude variable (0.11 mag in $U)$. He derived a period of 1.13254 days and used $30 \mathrm{Cyg}$ as his comparison star. Many of our FCAPT observations were apparently no good if we demanded that our $c h-c$ values remained constant. Using 9 FCAPT observations
HR 7721

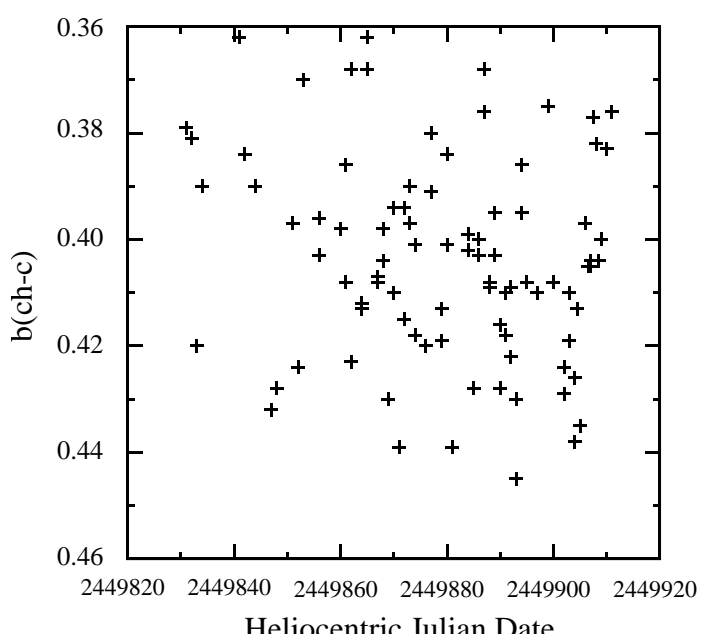

Fig. 3. The $b$ photometry of HR 7721 plotted as a function of Heliocentric Julian Date for the 1994-95 observing season 
in $1990-91,19$ in $1992-93,18$ in 1993-94, and 44 in 199495 a periodogram analysis showed that the most probable period was 8.5259 days. Comparison of our $y$ data with Winzer's $V$ values showed that there was a small phase shift. Thus the period was found to be $8.5297 \pm 0.0002$ days. As light maxima are sharper than the broad minima, we selected the zero epoch to fall within Winzer's observations, $2441618.86 \pm 0.02$. After the Hipparcos data (ESA 1997) become available (see Table 5), it was apparent that the comparison star HR 7721, a known spectroscopic binary, (Hoffleit 1982) was variable.

To improve the quality of our light curves and to attempt to find the nature of HR 7721's variability, we plotted our $v$ - $c$ data according to our period. Then we deleted values which were clearly in error. This left 21 FCAPT values in 1990-91, 39 in 1992-93, 39 in 1993-94, and 107 in 1994-95, numbers which are substantially greater than those used to find the period.

Figure 2 shows that the uvby light curves are approximately in phase. The $V$ observations of Winzer (closed circles) as normalized to the $y$ observation zero point overlay the $y$ FCAPT values (pluses). There are suggestions of structure in the light curve. The amplitude in $u$ is $0.13 \mathrm{mag}$, and in $v, b$, and $y 0.065 \mathrm{mag}$. Thus the energy distribution just above the Balmer jump remains relatively constant.

HR 7721 is a spectroscopic binary with a period of 7.18584 days (Hube \& Lowe 1980). When we used a Scargle periodogram on the $y(c h-c)$ data we found a period of either 1.10267 or 10.0733 days. Plotting the data against phases based on these two periods or the binary period showed mostly scatter. For 10.0733 days, we saw the suggestion of a modulation with about 0.1 magnitude of scatter. When we look only at one season of photometry, for some magnitudes we see suggestions of a semi-periodic variability, but not for others using these three different period. Figure 3 shows the $b$ photometry for the 1994-95 observing season. As HR 7721 is a B7 $V$ star, at least one of its components might be a slowly pulsating B star. Clearly this is a star which requires more study.

\section{Constancy of comparison and check stars from Hipparcos photometry}

Adelman (1998) advocated that astronomers who perform differential photometry check the observations of their comparison and check stars in the Hipparcos photometry database (ESA 1997). In this section, we do this for groups of B, A, and F stars that are on his observing program, or were observed, but their data has not yet been analyzed, with the Four College Automated Photoelectric Telescope. Table 5 contains this type of data as well as that for the variable stars when observed by Hipparcos: the mean magnitude, the standard error, and the amplitude taken to be the difference between the 95 th and 5 th percentile observations. Periods from the Hipparcos database are given.

In selecting comparison (c) and check (ch) stars, one tries to find non-variable stars close on the sky to the variable $(v)$ which are similar in magnitude and in color and also will be the only star in the photometer's aperture. Sometimes it is impossible to satisfy all of these requirements and either one must compromise or does not perform differential photometry of a given star. Most of the comparison and check stars were found by consulting the Bright Star Catalogue and its supplement (Hoffleit 1982; Hoffleit et al. 1983) and the rest from finding which stars other photometrists had used. In deciding which comparison and check stars were questionable, we considered how the Hipparcos standard errors grow as the stars get fainter. We have also included any original comparison and check stars indicated as oc and oh, respectively, which we have replaced as part of this exercise.

Acknowledgements. This work was supported in part by NSF grants AST-9115114 and 9528506 and in part by grants from The Citadel Development Foundation. We appreciate the continuing efforts of Louis J. Boyd, Robert J. Dukes, Jr., and George P. McCook to keep the FCAPT operating properly.

\section{References}

Adelman S.J., 1988, Baltic Astron. 1, 427

Babcock H.W., 1958, ApJS 3, 141

Brewer K.R.W., 1953, ApJ 118, 265

ESA, 1997, The Hipparcos and Tycho Catalogues, SP-1200

Gulliver A.F., Hube D.P., King R.W., Morris S.L., Hill G., 1985, AJ 90, 1334

Heck A., Maitzen H.M., Renson P., 1977, A\&A 54, 635

Heck A., Mathys G., Manfroid J., 1987, A\&AS 70, 33

Hensberge H., Maitzen H.M., Deridder G., et al., 1981, A\&AS 46,51

Hoffleit D., 1982, The Bright Star Catalogue, 4th edition. Yale University Observatory, New Haven, CT

Hoffleit D., Saladyga M., Wlasuk P., 1983, A Supplement to the Bright Star Catalogue, Yale University Observatory. New Haven, CT

Horne J.H., Baliunas S.L., 1986, ApJ 302, 757

Hube D.P., 1981, AJ 86, 1394

Hube D.P., Lowe A., 1980, JRASC 74, 342

Mathys G., Hubrig S., 1997, A\&AS 124, 475

Mathys G., Hubrig S., Landstreet J.D., Lanz T., Manfroid J., 1997, A\&AS 123, 353

Morris S.L., 1985, ApJ 295, 143

Scargle J.D., 1982, ApJ 263, 835

Winzer J.E., 1974, Ph. D. Dissertation, University of Toronto 